\title{
Crenças dos profissionais da Atenção Primária à Saúde de Juiz de Fora em relação à violência doméstica contra idosos ${ }^{1}$
}

\author{
Beliefs of primary health care professionals \\ of Juiz de Fora as regards domestic \\ violence against elderly
}

\author{
Lélio Moura LOURENÇO² \\ Daniela Cristina Belchior MOTA ${ }^{2}$ \\ Roberta Gonçalves CARVALHO² \\ Carla Ferreira de Paula GEBARA ${ }^{3}$ \\ Telmo Mota RONZANI ${ }^{2}$
}

\begin{abstract}
Resumo
Este estudo focaliza a violência doméstica contra idosos sob a perspectiva das crenças dos profissionais da Atenção Primária à Saúde de Juiz de Fora, Brasil. A amostra constituiu-se de 197 profissionais do Programa Saúde da Família. Realizaram-se análises de correlação entre as crenças dos profissionais de saúde e os tipos de violência doméstica e o grau de parentesco dos agressores, bem como da relação entre violência doméstica e consumo de álcool e drogas. Foi também observada a atuação dos profissionais de saúde diante da suspeita ou confirmação de violência e, ainda, a existência ou não de treinamento para esses profissionais. Os resultados apontaram que a atuação dos profissionais de saúde está restrita à pratica de encaminhamentos e que parte de suas crenças foram confirmadas pelos dados da literatura. Essa confirmação pode ter sido viabilizada pela experiência de contato dos profissionais com a realidade da violência doméstica. Potencialmente, os profissionais de saúde poderiam ter uma atuação voltada para a violência doméstica contra idosos.
\end{abstract}

Unitermos: Atenção primária à saúde. Crenças. Idosos. Violência doméstica.

\footnotetext{
Abstract

This study characterized domestic violence against the elderly, from the perspective of beliefs of primary care professionals of Juiz de Fora, Minas Gerais, Brazil. The sample consisted of 197 professionals from the Family Health Program. Data was analyzed using analysis of correlation between the beliefs of health professionals and the types of violence, degree of relationship of the perpetrators, correlation between

$\operatorname{crv}$

1 Apoio: Fundação de Amparo à Pesquisa de Minas Gerais. Projeto de Iniciação Científica Processo n 024.08.0077.

2 Universidade Federal de Juiz de Fora, Faculdade de Psicologia. R. José Lourenço Kelmer, s/n., Campus Universitário, Martelos, 36036-900, Juiz de Fora, MG, Brasil. Correspondência para/Correspondence to: L.M. LOURENÇO. E-mail: <leliomlourenco@yahoo.com.br>.

3 Universidade Federal de São Paulo, Departamento de Psicobiologia. São Paulo, SP, Brasil.

Agradecimento: aos membros do grupo de pesquisa "Crenças dos Profissionais de Saúde sobre Violência Doméstica".
} 
domestic violence and alcohol and drug consumption. In addition the performance of health professionals on suspicion or confirmation of violence was observed, and whether or not they were receiving training. The Results showed that the performance of health professionals was restricted to referral practices and that some of their beliefs were confirmed by literature data. This confirmation may have been enabled by the professional's experience of contact with the reality of domestic violence. Potentially, these health professionals could take action directed towards controling/preventing? domestic violence against the elderly.

Uniterms: Primary health care. Beliefs. Elderly. Domestic violence.

Nas últimas décadas, o Brasil vem passando por um intenso processo de envelhecimento populacional. O número de indivíduos com 60 anos ou mais representa 9,3\% da população brasileira, conforme dados do Departamento de Informática do Sistema Único de Saúde (2009). Em relação ao mundo, dados da Organização das Nações Unidas (ONU) revelam que os idosos já atingiram o percentual de 12,0\% da população total, conforme o Instituto Brasileiro de Geografia e Estatística (2002). O aumento expressivo da população idosa nos últimos anos no Brasil e no mundo tem sua procedência a partir das transformações sociais, econômicas e políticas que determinaram grandes inovações técnico-científicas, associadas à melhoria da vida da população em geral (Santos, Silva, Carvalho \& Menezes, 2007).

Em conjunto com as modificações apresentadas pelo aumento da expectativa de vida da população coexistem importantes transformações relacionadas com a estrutura familiar, como o aumento das separações, divórcios, novas uniões, vivências intergeracionais, instabilidade financeira e participação crescente das mulheres no mercado de trabalho. Essas transformações têm gerado abalos na estrutura familiar, conduzindo a uma deterioração da qualidade de vida e contribuindo para o aumento de relações intrafamiliares conflituosas (Organização Mundial de Saúde, 2002; Minayo, 2003, 2006). De outro lado, estudos apontam que a qualidade de vida de idosos está associada a um bom relacionamento com a família (Irigaray \& Trentini, 2009). Nesse contexto, o fenômeno da violência ganha um relevante enfoque na dinâmica familiar, na qual a violência domés-

3 tica destaca-se como um expressivo fator de risco para ¿ a saúde emocional, psíquica e social das pessoas idosas.

O Ministério da Saúde Brasileiro (Brasil, 2001, p.28) define o maltrato da seguinte forma:"o maltrato ao idoso é um ato (único ou repetido) ou omissão que lhe cause dano ou aflição e que se produz em qualquer relação na qual exista expectativa de confiança". Considera-se 428 que tal ato se refere a várias dimensões de abuso: (a) abuso psicológico, envolvendo agressões verbais ou gestuais, com objetivo de aterrorizar, rejeitar ou humilhar a vítima; (b) abuso físico, referente ao uso da força física, produtor de injúria, ferida, dor ou incapacidade; (c) abuso econômico, concernente à exploração indevida dos recursos financeiros e patrimoniais do idoso; (d) abuso sexual, considerado o ato ou jogo sexual, em uma relação hetero ou homossexual, visando a práticas eróticas por meio de aliciamento, violência física ou ameaças; (e) abandono, manifestado pela ausência ou deserção dos responsáveis junto ao idoso e (f) negligência, com recusa ou omissão dos cuidados devidos e necessários aos idosos por parte dos seus responsáveis, e/ou autonegligência, concernente à conduta de ameaça à própria saúde ou segurança, com a recusa ou o fracasso do idoso em prover-se de um cuidado adequado.

Devido aos agravos que causa à saúde da pessoa idosa, a violência doméstica é considerada atualmente como um sério problema de saúde pública, que onera o sistema de saúde com gastos hospitalares, eleva os níveis de mortalidade, reduz anos de vida produtiva e pode ocasionar sequelas irreversíveis para as pessoas. Algumas pesquisas encontraram relevantes dados que demonstram grande incidência de casos de agressão contra idosos em diversos países desenvolvidos, como Estados Unidos, Canadá, Reino Unido, os quais fazem vultosos investimentos para eliminar o problema, configurando um assunto de âmbito mundial (Bonnie Brandl \& Cook-Daniels, 2002; Sanches, Lebrão \& Duarte, 2008).

Vale destacar ainda fatores como o uso de álcool e outras drogas, os quais atuam como um forte agravante no conflito intrafamiliar. Estudos revelam um alto índice de correlação entre o abuso de substâncias psicoativas e a violência doméstica, indicando que seu uso deve ser levado em consideração na dinâmica relacional do idoso com os familiares (Noto, Fonseca, Silva \& Galduròz, 2004; Souza, Meira, Neri, Silva \& Gonçalves, 2004).

Por outro lado, essa temática comporta uma análise psicossocial a partir do estudo das crenças dos 
profissionais de saúde, as quais apresentam um papel considerável no entendimento de suas atitudes, valores e comportamentos. Entende-se por crença "uma proposição que, na sua formulação mais simples, afirma ou nega uma relação entre dois aspectos concretos ou abstratos ou entre um objeto e um possível atributo deste" (Krüger, 1995, p.27). As crenças são organizadas em sistemas ou conjuntos logicamente estruturados, sendo capazes de ativar motivações e, portanto, condutas sociais, influenciando por essa via processos coletivos (Krüger, 1995). Krüger (1993) destaca ainda que, no plano subjetivo, as crenças se encontram articuladas a valores e a atitudes, sendo, portanto, elementos de representação mental essencialmente abstratos, oriundos de experiências individuais e coletivas. Rokeach (1981) faz uma analogia, apontando que elas estão organizadas como sistemas arquitetônicos, mas não necessariamente de forma lógica. Aponta que as crenças possuem propriedades estruturais descritíveis e mensuráveis que, por sua vez, possuem consequências comportamentais observáveis.

Desse modo, o estudo das crenças dos profissionais de saúde é importante para o entendimento das práticas dos mesmos nos casos de violência doméstica contra a população idosa do município. Ressalta-se que o Sistema Único de Saúde (SUS) tem investido na ampliação do atendimento por meio da Atenção Primária à Saúde (APS) e do Programa Saúde da Família (PSF), considerados, por muitos autores, potenciais mecanismos de identificação, atendimento e prevenção contra a violência doméstica. Os profissionais da APS possuem contato direto com a maioria da população idosa, o que possibilita acesso aos casos de violência doméstica não registrados pelos órgãos competentes (Sanches et al., 2008; Silvestre \& Costa Neto, 2003).

O objetivo do presente artigo é caracterizar a violência doméstica contra o idoso, correlacionada ou não ao uso de álcool e/ou outras substâncias psicoativas, sob a perspectiva das crenças dos profissionais da equipe mínima do Programa Saúde da Família do município de Juiz de Fora (MG).

\section{Método}

O estudo foi realizado no município brasileiro de Juiz de Fora, situado no Estado de Minas Gerais. 0 município possui aproximadamente 526709 habitantes, sendo que 12,5\% da população têm 60 anos de idade ou mais. Atualmente, a APS de Juiz de Fora é composta por 48 Unidades Básicas de Saúde (UBS), com 511 profissionais atuantes no Programa Saúde da Família (Departamento de Informação do Sistema Único de Saúde, 2009).

\section{Participantes}

Para este estudo, foram selecionados 195 profissionais de saúde, representando todas as UBS da zona urbana de Juiz de Fora que possuíssem o PSF. Para compor a amostra foi escolhida aleatoriamente uma equipe mínima de cada UBS, abrangendo várias áreas de cobertura do PSF. Segundo o Ministério da Saúde Brasileiro (Brasil, 2004), a equipe mínima do PSF é composta por um médico de família, um enfermeiro, um auxiliar de enfermagem e seis Agentes Comunitários de Saúde (ACS). Na Tabela 1 é possível visualizar a descrição dos participantes deste estudo, considerando a categoria profissional, o sexo e o tempo de trabalho no PSF. Como pode ser observado, a maioria dos participantes são ACS (69,0\%). Ainda pode ser verificado que a maior parte dos profissionais de saúde é do sexo feminino $(84,7 \%)$, sendo que somente $8,1 \%$ ainda não completou 1 ano de trabalho no PSF.

Para a coleta de dados foi necessário agendar um horário com o gerente de cada UBS, a fim de solicitar a autorização para que os profissionais de saúde participassem da pesquisa. O questionário da pesquisa foi aplicado no primeiro semestre de 2007, no horário de trabalho dos profissionais e conforme a conveniência das equipes de PSF.

\section{Instrumentos}

Os profissionais de saúde foram convidados a responder voluntariamente a um questionário autoaplicável, individual e sigiloso.

O questionário objetivou avaliar as crenças dos profissionais de saúde com relação à violência doméstica contra idosos, através de variáveis estruturadas e não estruturadas. As variáveis estruturadas avaliaram o tipo de violência doméstica mais frequentemente detectado; o grau de parentesco dos agressores; as providências tomadas diante de suspeita ou confir- 
Tabela 1. Descrição dos participantes do estudo. Frequência e Porcentagem. Juiz de Fora (MG), 2009.

\begin{tabular}{|c|c|c|c|c|c|c|c|c|c|c|}
\hline \multirow{3}{*}{ Profissão } & \multicolumn{4}{|c|}{ Sexo } & \multicolumn{6}{|c|}{ Tempo de serviço } \\
\hline & \multicolumn{2}{|c|}{ Feminino } & \multicolumn{2}{|c|}{ Masculino } & \multicolumn{2}{|c|}{ Até 1 ano } & \multicolumn{2}{|c|}{$1-5$ anos } & \multicolumn{2}{|c|}{ Mas de 5 anos } \\
\hline & $n$ & $\%$ & $n$ & $\%$ & $n$ & $\%$ & $n$ & $\%$ & $\mathrm{n}$ & $\%$ \\
\hline Médico & 12 & 6,1 & 7 & 3,6 & 1 & 0,5 & 6 & 3,0 & 12 & 6,1 \\
\hline Enfermeiro & 19 & 9,6 & 1 & 0,5 & 4 & 2,0 & 5 & 2,5 & 11 & 5,6 \\
\hline Auxiliar de enfermagem & 19 & 9,6 & 1 & 0,6 & 0 & 0,0 & 14 & 7,1 & 5 & 2,5 \\
\hline ACS & 117 & 59,4 & 19 & 9,6 & 11 & 5,6 & 58 & 29,4 & 69 & 35,0 \\
\hline Total & 167 & 84,7 & 28 & 14,3 & 16 & 8,1 & 83 & 42,0 & 97 & 49,2 \\
\hline
\end{tabular}

ACS: Agentes Comunitários de Saúde.

mação de ato violento; e a correlação entre a violência doméstica e o consumo de álcool e/ou outras substâncias psicoativas. Já as três variáveis não estruturadas avaliaram as crenças quanto à ocorrência de diferença entre violência doméstica contra crianças/adolescentes e violência contra idosos; as crenças quanto ao aumento da ocorrência de violência doméstica contra idosos nos últimos anos; e a oferta ou não de treinamento/informações, aos profissionais de saúde, a respeito da violência contra idosos.

Este questionário fora elaborado anteriormente pelo grupo de pesquisadores, em um estudo mais amplo que verificara as crenças dos profissionais da área jurídica, de educação e de saúde da atenção terciária, a respeito da violência doméstica no município de Juiz de Fora. Posteriormente, o questionário foi adaptado para a realização deste estudo, voltado a profissionais da APS. O instrumento incluiu também a avaliação das crenças em relação à violência doméstica contra crianças e adolescentes.

O protocolo de pesquisa foi aprovado pelo Comitê de Ética em Pesquisa com Seres Humanos da Universidade Federal de Juiz de Fora com emissão do parecer (102/2006). Todos os profissionais de saúde que participaram do estudo foram informados do objetivo da pesquisa, bem como resguardada a identidade e o sigilo das informações. Após esses esclarecimentos, cada participante foi solicitado a ler o Termo de Consentimento Livre Esclarecido e a assiná-lo, caso concordasse em colaborar com a pesquisa.

Os dados provenientes das variáveis estruturadas do questionário foram organizados e analisados no software Statistical Package for the Social Sciences (SPSS),

430 versão 15.0. Foram utilizadas estatísticas descritivas como frequência (n) e frequência relativa (\%). A análise de associação entre as crenças dos profis-sionais de saúde foi baseada no Qui-quadrado de Pearson $\left(\chi^{2}\right)$, no Odds Ratio (OR) e seus intervalos de confiança. Optou-se pela utilização da correção do teste exato de Fisher quando as propriedades do teste de Qui-quadrado não fossem satisfeitas.

A partir desse processo de análise, buscou-se o melhor modelo explicativo para o conjunto dos dados encontrados, avaliando-se o grau de efeito das variáveis umas às outras. Por intermédio da estimação e análise dos OR, foram encontradas as magnitudes de associação entre as crenças dos profissionais de saúde quanto aos tipos de violência contra idosos e a caracterização desses tipos de violência como doméstica, bem como a associação entre a tipificação de violência e o grau de parentesco dos agressores. Nas análises estatísticas estabeleceu-se um nível de significância de 5\% $(p<0,05)$.

Os dados obtidos com a parte não estruturada do questionário foram organizados e submetidos à técnica de análise de conteúdo do tipo estrutural e temática (Bardin, 1977).

\section{Resultados}

\section{Tipo de violência contra idosos e sua caracterização como violência doméstica}

Dos 195 participantes, 52,5\% afirmaram já ter detectado algum tipo de violência contra idosos. Quanto à caracterização dessa violência como "doméstica", $58,1 \%$ dos participantes assim a classificam, enquanto 18,7\% acreditam que ela não se enquadre nessa cate- 
goria. A maior parte dos participantes (76,8\%) apontou ter conhecimento sobre os tipos de violência doméstica.

A Tabela 2 indica que os tipos de violência doméstica mais frequentes contra idosos são a violência financeira (80,3\%) e o abandono (75,7\%). Conforme pode ser observado, há uma associação significativa entre os profissionais de saúde caracterizarem a violência contra idosos como violência doméstica e indicarem a negligência como tipo de violência. Os resultados demonstram que, quando a violência contra idosos é caracterizada como doméstica, há uma chance maior de esta violência ser tipificada como negligência $(O R=2,24$; $1,01-4,96 ; p=0,04)$. Destaca-se que tal associação não foi observada de modo significativo para nenhum outro tipo de violência considerado neste estudo.

\section{Tipo de violência contra idosos e grau de parentesco dos agressores}

Em relação ao grau de parentesco dos agressores, os profissionais de saúde apontaram os filhos $(73,2 \%)$ como sendo os agressores de idosos mais frequentes na violência intrafamiliar. Os outros agressores apontados foram os netos (39,4\%), os cuidadores não parentes (34,5\%), os outros parentes $(23,7 \%)$ e os cônjuges (22,7\%).

Nas Tabelas 3 e 4 são apresentadas as correlações entre as crenças dos profissionais de saúde no que se refere à associação entre os tipos de violência e o grau de parentesco dos agressores. Com exceção da violência sexual, há uma tendência de correlacionar os filhos como os familiares que apresentam risco significativo de serem os agressores na violência doméstica contra idosos em todos os outros tipos de violência. Quanto à magnitude desse risco, os profissionais de saúde acreditam que o risco de os filhos serem os agressores é ainda seis ve-zes maior se o tipo de violência doméstica apontado for o abandono $(\mathrm{OR}=6,10 ; 3,01$ $12,36 ; p=0,001)$ e cinco vezes maior quando a violência for financeira (OR=5,03;2,44-10;36; $p=0,001)$.

Para os profissionais de saúde que indicaram os netos como sendo os agressores dos idosos, mostrouse uma associação positiva somente em relação às crenças de ocorrência de violência financeira $(O R=3,27$; $1,47-7,27 ; p=0,003)$ e psicológica $(\mathrm{OR}=2,85 ; 1,58-5,16$; $p=0,001)$.

Segundo as crenças dos profissionais de saúde, pode ser estimado que o único grau de parentesco que apresenta risco de magnitude significativa para a ocorrência de todos os tipos de violência são os cônjuges. De acordo com os resultados, há um maior risco de os cônjuges serem agressores na violência tipificada como abandono (OR=4, 97; 1,68-14,67; $p=0,002)$.

Ao considerarem os cuidadores como sendo os agressores, verificou-se que há uma correlação significativa com todos os tipos de violência, exceto a sexual. Destaca-se que a magnitude dessa associação é maior

Tabela 2. Correlação entre o tipo de violência e a caracterização da violência contra idosos como Violência Doméstica (VD). Frequência, Porcentagem, Teste de Qui-quadrado, OR, intervalos de confiança e valores de p. Juiz de Fora (MG), 2009.

\begin{tabular}{|c|c|c|c|c|c|c|c|c|c|c|}
\hline \multirow{3}{*}{ Tipo de violência } & & \multicolumn{6}{|c|}{ Caracterização como VD } & \multirow{3}{*}{$\chi^{2}$} & \multirow{3}{*}{ OR, IC (95\%) } & \multirow{3}{*}{$p$} \\
\hline & & \multicolumn{2}{|c|}{ Não } & \multicolumn{2}{|c|}{$\operatorname{sim}$} & \multicolumn{2}{|c|}{ Total } & & & \\
\hline & & $n$ & $\%$ & $n$ & $\%$ & $n$ & $\%$ & & & \\
\hline \multirow{2}{*}{ Financeira } & Não & 7 & 4,6 & 23 & 15,1 & 30 & 19,7 & 0,21 & $0,933(0,36-2,39)$ & 0,880 \\
\hline & $\operatorname{Sim}$ & 30 & 19,7 & 92 & 60,5 & 22 & 80,3 & & & \\
\hline \multirow{2}{*}{ Negligência } & Não & 26 & 17,1 & 59 & 38,8 & 85 & 55,9 & 4,00 & $2,240(1,01-4,96)$ & $0,043^{*}$ \\
\hline & Sim & 11 & 7,2 & 56 & 36,8 & 67 & 44,1 & & & \\
\hline \multirow{2}{*}{ Abandono } & Não & 10 & 6,6 & 27 & 17,8 & 37 & 24,3 & 0,19 & $1,200(0,51-2,80)$ & 0,660 \\
\hline & Sim & 27 & 17,8 & 88 & 57,9 & 115 & 75,7 & & & \\
\hline \multirow{2}{*}{ Psicológica } & Não & 18 & 11,8 & 60 & 39,5 & 78 & 51,3 & 0,13 & $0,860(0,41-1,82)$ & 0,700 \\
\hline & Sim & 19 & 12,5 & 55 & 36,2 & 74 & 48,7 & & & \\
\hline \multirow{2}{*}{ Física } & Não & 25 & 16,4 & 66 & 43,4 & 91 & 59,9 & 1,20 & $1,540(0,70-3,37)$ & 0,270 \\
\hline & Sim & 12 & 7,9 & 49 & 32,2 & 61 & 40,1 & & & \\
\hline \multirow{2}{*}{ Sexual } & Não & 37 & 24,3 & 106 & 69,7 & 143 & 94,1 & 3,07 & $1,080(1,02-1,14)$ & 0,114 \\
\hline & Sim & 0 & 0,0 & 9 & 5,9 & 9 & 5,9 & & & \\
\hline
\end{tabular}


no caso da violência financeira $(O R=5,58 ; 2,08$ - 14 96; $p=0,001)$.

É possível ressaltar, ainda, que os outros parentes apresentem associações significativas se os tipos de violência considerados forem o abandono, a violência financeira e a negligência, sendo maior o risco estimado no caso do abandono (OR=4,03; 1,05-10,84; $p=0,003)$.

Tais resultados permitem observar que as crenças dos profissionais de saúde indicam um risco maior de ocorrer a violência financeira ou o abandono para todos os graus de parentesco de agressores considerados no questionário (Tabelas 3 e 4).

\section{Violência doméstica contra idosos e uso de álcool e outras drogas}

Na Figura 1 são apresentadas as crenças dos profissionais de saúde quanto à relação entre a violência doméstica contra idosos e o uso de álcool e outras drogas. Comparando os resultados apresentados, é possível conferir que as outras drogas $(60,2 \%)$ são mais frequentemente apontadas por "tornar a pessoa mais violenta" do que o uso de álcool (53,0\%). No entanto, se considerada a categoria "estimula a violência somente em pessoas com tendência", o uso de álcool $(37,0 \%)$ é mais frequentemente apontado pelos profissionais de saúde do que o uso de outras drogas (32,6\%).

\section{Atuação dos profissionais de saúde}

Questionados sobre quais as providências que deveriam ser tomadas ao se detectarem casos de violência doméstica contra idosos, as alternativas mais citadas pelos profissionais foram: encaminhamento ao Serviço Social (51,5\%), ao Centro de Proteção e Defesa aos Direitos da Pessoa Idosa (CEDDI) (44,4\%), ao Conselho Municipal do Idoso (42,9\%) e à delegacia (10,6\%).

\section{Dados qualitativos}

Um total de 135 profissionais de saúde (45,1\%) acredita haver diferença entre a violência doméstica contra crianças e adolescentes e a violência doméstica

Tabela 3. Correlação entre o tipo de violência contra idosos e o grau de parentesco do agressor. Porcentagem, Teste Qui-quadrado, OR e valores de $p$. Juiz de Fora (MG), 2009

\begin{tabular}{|c|c|c|c|c|c|c|c|c|c|c|c|c|}
\hline \multirow{2}{*}{ Parentesco } & \multicolumn{4}{|c|}{ Financeira } & \multicolumn{4}{|c|}{ Negligência } & \multicolumn{4}{|c|}{ Abandono } \\
\hline & $\%$ & $\chi^{2}$ & OR & $p$ & $\%$ & $\chi^{2}$ & OR & $p$ & $\%$ & $\chi^{2}$ & OR & $p$ \\
\hline Cônjuge & 21,0 & 6,26 & 3,72 & $0,012^{*}$ & 14,4 & 6,90 & 2,47 & $0,009^{*}$ & 21,0 & 9,88 & 4,97 & $0,002^{*}$ \\
\hline Filho & 63,6 & 21,13 & 5,03 & $0,001^{*}$ & 37,4 & 6,21 & 2,36 & $0,013^{*}$ & 61,5 & 28,21 & 6,10 & $0,001^{*}$ \\
\hline Neto & 35,4 & 9,04 & 3,27 & $0,003^{*}$ & 20,5 & 1,98 & 1,51 & 0,150 & 31,3 & 1,90 & 1,50 & 0,160 \\
\hline Outros & 21,5 & 5,04 & 3,00 & $0,025^{*}$ & 16,4 & 13,18 & 3,50 & $0,001^{*}$ & 21,5 & 8,56 & 4,03 & $0,003^{*}$ \\
\hline Cuidadores & 32,3 & 3,82 & 5,58 & $0,001^{*}$ & 20,5 & 7,90 & 2,35 & $0,005^{*}$ & 30,3 & 10,25 & 3,47 & $0,001^{*}$ \\
\hline Sem condições para responder & 2,6 & 27,98 & 0,082 & $0,001^{*}$ & 2,6 & 2,41 & 0,43 & 0,140 & 4,1 & 8,06 & 0,25 & $0,007^{*}$ \\
\hline
\end{tabular}

${ }^{*} p<0,05$. OR: Odds Ratio.

Tabela 4. Correlação entre o tipo de violência contra idosos e o grau de parentesco do agressor. Porcentagem, Teste Qui-quadrado, OR e valores de p. Juiz de Fora (MG), 2009.

\begin{tabular}{|c|c|c|c|c|c|c|c|c|c|c|c|c|}
\hline \multirow{2}{*}{ Parentesco } & \multicolumn{4}{|c|}{ Psicológica } & \multicolumn{4}{|c|}{ Física } & \multicolumn{4}{|c|}{ Sexual } \\
\hline & $\%$ & $\chi^{2}$ & OR & $p$ & $\%$ & $\chi^{2}$ & OR & $p$ & $\%$ & $\chi^{2}$ & OR & $p$ \\
\hline Cônjuge & 16,4 & 14,66 & 3,90 & $0,001^{*}$ & 13,3 & 9,76 & 2,90 & $0,002^{*}$ & 3,1 & 5,150 & 3,66 & $0,023^{*}$ \\
\hline Filho & 37,9 & 5,42 & 2,21 & $0,020^{*}$ & 32,8 & 9,19 & 3,16 & $0,002^{*}$ & 4,6 & 0,004 & 1,04 & 0,950 \\
\hline Neto & 24,6 & 12,98 & 2,85 & $0,001^{*}$ & 19,0 & 4,96 & 1,95 & 0,026 & 3,6 & 1,740 & 2,18 & 0,220 \\
\hline Outros & 0,0 & 1,73 & 0,98 & 0,180 & 0,0 & 1,23 & 0,98 & 0,26 & 0,0 & 0,130 & 0,98 & 1,000 \\
\hline Cuidadores & 24,1 & 22,15 & 4,37 & $0,001^{*}$ & 21,0 & 22,14 & 4,32 & $0,001^{*}$ & 2,6 & 0,240 & 1,34 & 0,620 \\
\hline Sem condições para responder & 1,5 & 6,93 & 0,20 & $0,008^{*}$ & 1,0 & 6,06 & 0,18 & $0,014^{*}$ & 1,0 & 0,890 & 1,33 & 0,630 \\
\hline
\end{tabular}

432

* $p<0,05$. OR: Odds Ratio 


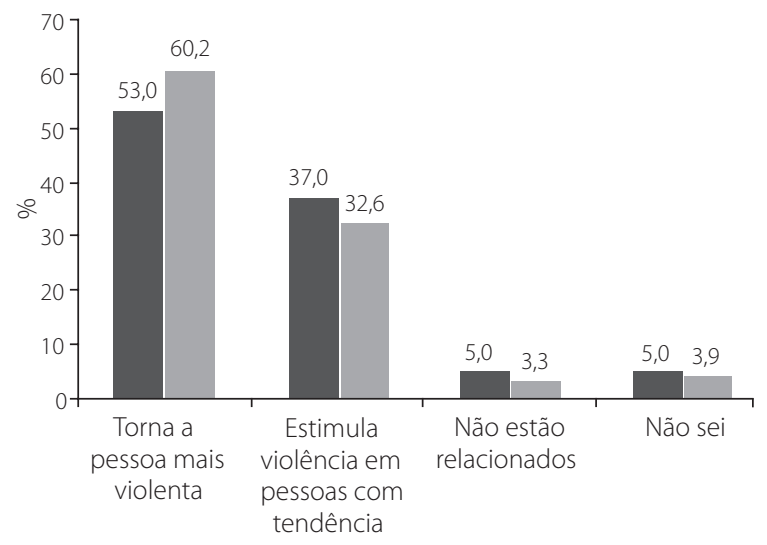

álcool Outras drogas

Figura 1. Crenças dos profissionais de saúde quanto à relação entre a violência domestica contra idosos e o uso de álcool e outras drogas. Juiz de Fora (MG), 2009.

contra idosos. Entre estes, 51,0\% são médicos, enfermeiros e auxiliares de enfermagem, enquanto 49,0\% são ACS.

Verificou-se que $83,5 \%$ dos profissionais de saúde acreditam que houve um aumento na ocorrência dos casos de violência intrafamiliar contra idosos nos últimos anos. Os fatores apontados como determinantes para o aumento da ocorrência dos casos de violência doméstica foram as questões socioeconômicas (50,0\%); os valores sociais, como sensação de inutilidade e falta de solidariedade, amor, respeito e paciência (20,0\%); as dificuldades para lidar com o envelhecimento, como a ocorrência de doenças nessa idade e o tempo requerido para esses cuidados (15,0\%); o aumento da expectativa de vida (10,0\%); a correlação com álcool e drogas (6,0\%); e outros, como a falta de conhecimento sobre o envelhecimento (7,0\%).

Quanto ao treinamento dos profissionais de saúde em relação à violência doméstica, 80,7\% dos respondentes afirmaram ter feito curso de capacitação, enquanto 4,4\% adquiriram conhecimento exclusivamente através de informações.

\section{Discussão}

A convivência intergeracional constitui um substrato para a construção do processo de socialização, aprendizagem e desenvolvimento saudável na vida dos indivíduos. Entretanto, a família tem encontrado diversas dificuldades para cumprir suas funções psicossociais, o que em muitos casos favorece a ocorrência de violência (Minayo, 2006; Santos et al., 2007).

A literatura aponta que a maior parte da violência sofrida pelos idosos acontece no lar (Minayo, 2006; Souza, Ribeiro, Atie, Souza \& Marques, 2008). Algumas pesquisas sobre levantamentos de denúncias em órgãos especializados mostraram a violência financeira, outras, o abandono ou a negligência como as mais frequentes, sendo que a maior ocorrência está entre esses três tipos de violência (Porto \& Koller, 2006; Sanches et al., 2008; Souza, Freitas \& Queiroz, 2007).

Corroborando os dados de pesquisas realizadas em delegacias e órgãos de proteção ao idoso, os resultados do presente estudo revelaram que, segundo as crenças dos profissionais de saúde, o abuso financeiro e o abandono são os tipos de violência mais frequentes na comunidade. Ainda que as análises descritivas tenham apontado tais resultados, a negligência foi o único tipo que apresentou correlação significativa com as crenças dos profissionais quanto à ocorrência de violência doméstica contra idosos. Destaca-se que os idosos são vitimados simultaneamente com diversas formas de violência, e que a concomitância das agressões traz diversos agravos para a saúde, ocasionando múltiplos transtornos psicossociais para a pessoa idosa (Machado \& Queiroz, 2002; Moraes, Apratto Júnior \& Reichenheim, 2008). Assim, as crenças dos profissionais de saúde podem ter se correlacionado unicamente com a negligência tendo em vista a dificuldade dos mesmos em detectar e diferenciar os tipos de violência.

No que concerne à violência sexual, a literatura destaca que os idosos também são vítimas desse abuso, mas que esse é um tipo de violência menos frequente para os idosos (Pasinato, Camarano \& Machado, 2006). Neste estudo, as crenças apontaram o que comumente se atribui ao idoso, denotando que tal tipo de violência quase não ocorre.

Estudos têm demonstrado também que os tipos de violência estão vinculados ao grau de parentesco do agressor com a vítima, sendo os filhos associados à violência financeira e à negligência; os netos, ao abuso financeiro e ao abandono; e os cônjuges ao abandono e ao abuso sexual (Espíndola \& Blay, 2007; Pasianato 
et al. 2004). Dados relevantes para a compreensão da dinâmica familiar ressaltam que ser mulher e ser idosa são fatores consideráveis para ser vítima de violência doméstica, sendo o cônjuge um dos principais agressores. Nesse caso, há uma tendência de que o homem idoso possa representar um duplo papel, sendo o agressor e ao mesmo tempo o agredido pelos outros familiares, principalmente filhos e netos (Porto \& Koller, 2006).

A ênfase, em parte das crenças dos profissionais de saúde, na violência financeira, correlacionada ao parentesco de primeiro e segundo grau (filhos e netos), pode estar associada a fatores de ordem socioeconômica. A condição financeira dos idosos - como principal provedor da família e, às vezes, único - pode ser considerada um fator de risco para a ocorrência dos conflitos familiares. No caso brasileiro, ressalta-se a significativa contribuição da renda dos idosos em seus lares, por meio da aposentadoria, de modo que dificuldades socioeconômicas podem gerar uma relação conflituosa entre os familiares (Souza et al., 2004).

A respeito da diferença entre a violência infanto-juvenil e a violência contra idosos, a maioria dos profissionais não apontou diferenças entre os abusos. Embora ambas as violências aconteçam no âmbito doméstico por pessoas que tenham algum vínculo afetivo, as motivações, as formas, os agressores e as relações conflituosas são dispostos por diferentes mecanismos dentro da dinâmica familiar.

Outro aspecto que vem sendo discutido nas pesquisas relaciona-se à associação entre violência e abuso de substâncias psicoativas. A incidência de violência demonstra-se maior em abusadores de substâncias psicoativas na maioria das sociedades e culturas, e presente nos diferentes grupos econômicos (Day et al., 2003; Souza et al., 2007). Ao investigarem a associação entre a ocorrência de violência e o consumo de substâncias psicoativas, estudos desta-cam que essa correlação é ainda mais forte no caso do abuso de álcool (HaggårdGrann, Hallqvist, Langström \& Moller, 2006; Noto et al., 2004). Diversos estudos demonstram que os índices de violência tendem a aumentar sistematicamente quando ocorre um aumento no padrão de consumo de álcool da população (Babor, 2003; Bye, 2007; Haggård-Grann et al., 2006). No caso da ocorrência de violência doméstica, Brito, Zanetta, Mendonça, Barison e Andrade (2005) demonstraram que 32\% das famílias afirmaram que o 434 álcool é um fator desencadeante da violência e 9\% afirmaram que a ocorrência de violência está associada às drogas ilícitas. Portanto, há uma incompatibilidade entre essas evidências e as crenças dos profissionais de saúde observadas neste estudo, na medida em que estes apontaram que as drogas ilícitas tornam a pessoa mais violenta.

De um modo geral, os principais resultados deste estudo ratificaram os achados de outras pesquisas, como a frequência dos tipos de violência doméstica e os principais agressores apontados, ainda que essa tendência não tenha sido observada em relação às substâncias psicoativas e em relação à diferenciação com a violência contra crianças e adolescentes. A avaliação das crenças dos profissionais de saúde demonstrou que estes parecem ter conhecimento sobre fatores importantes na ocorrência da violência doméstica contra idosos.

Destacam-se algumas considerações quanto ao processo de formação das crenças. Constituindo o sistema cognitivo, as crenças estão funcionalmente integradas às atitudes e aos valores, e são alteradas quando qualquer parte desse sistema sofre alguma modificação, podendo, inclusive despertar a mudança comportamental (Krüger, 1995). Tais alterações podem se dar através de propriedades cognitivas, ou seja, podem ser influenciadas por informações e treinamentos oferecidos aos profissionais, como podem também se dar através da experiência, ou seja, do contato com os casos de violência doméstica.

Sendo a aquisição de informações um fator que possibilita a mudança de crenças e, por conseguinte, de atitudes, sugere-se que a ocorrência de treinamentos pode ter contribuído para que parte das crenças dos profissionais de saúde fosse convergente com os dados encontrados nas pesquisas (Rokeach, 1981). Assim, uma limitação deste estudo é o fato de que tais treinamentos possam ter operado uma mudança nas crenças dos profissionais de saúde.

De outro lado, porém, considera-se que a promoção de cursos de capacitação para os profissionais de saúde - possibilitando aperfeiçoamento de práticas e mudança de crenças disfuncionais, como as relacionadas às substâncias psicoativas - pode ser incorporada à equipe do PSF, de forma continuada e de acordo com as necessidades de cada população adstrita, com a finalidade de atuarem na detecção precoce, bem como na interrupção dos casos de violência já instalados. 
Embora os profissionais ainda não executem ações de cuidados específicos e eficientes para os casos de violência contra idosos, a coincidência entre parte de suas crenças e os dados da literatura pode estar associada a seu contato com a violência doméstica contra idosos, em sua rotina de trabalho. Ressalta-se o papel dos profissionais da APS como aqueles que apresentam maior inserção comunitária e proximidade com a população. Isso possibilita que eles tenham uma atuação voltada para a violência doméstica, não só na prevenção, mas também como elo com a comunidade e os órgãos de apoio ao idoso, realizando o trabalho integrado com a rede, nos casos de notificação.

Contudo, este estudo demonstrou também que a atuação desses profissionais está restrita às praticas de encaminhamento para o assistente social. Os profissionais de saúde permanecem com um enfoque curativo, e não há uma ênfase na abordagem preventiva de atos violentos. Tendo em vista que a APS atinge a maior parte da população e que os profissionais de saúde demonstram, através de suas crenças, ter contato com a realidade da violência doméstica, observa-se que a APS pode ser a instância ideal para a identificação dos fatores de risco ao idoso, fazendo redimensionar a atuação dos profissionais de saúde frente ao problema.

\section{Considerações Finais}

A caracterização das crenças dos profissionais de saúde quanto à violência doméstica contra idosos demonstrou que eles parecem ter conhecimento sobre fatores importantes na ocorrência da violência, uma vez que a maior parte de suas crenças foi confirmada pelos dados de pesquisas. Considera-se que esta convergência entre as crenças dos profissionais e dos dados da literatura pode ter sido viabilizada pela experiência de contato desses profissionais com a realidade da violência doméstica contra idosos. Isso sugere que, potencialmente, tais profissionais de saúde podem ter uma atuação voltada para a violência doméstica contra idosos.

\section{Referências}

Bardin, L. (1977). Análise de conteúdo. Lisboa: Edições 70.
Babor, T. (2003). Alcohol: no ordinary, no commodity. Research and public policy. New York: Oxford University Press.

Bonnie Brandl, M. S. W., \& Cook-Daniels, L. (2002). Domestic abuse in later life: abusers. Journal of Elder Abuse and Neglect, 5 (4), 77-93.

Brasil. Ministério da Saúde. (2001, 18 de maio). Portaria GM/ MS no 737 de 16 de maio de 2001. Política nacional de redução da morbimortalidade por acidentes e violência. Diário Oficial da União, Seção I, n. 96.

Brasil. Ministério da Saúde. (2004). Atenção básica - PSF saúde da família. Recuperado em julho 15, 2009, disponível em <http://dab.saude.gov.br/>.

Brito, A. M., Zanetta, D. M. T, Mendonça, R. C. V., Barison, S. Z. P. \& Andrade, V. A. G. (2005). Violência doméstica contra crianças e adolescentes: estudo de um programa de intervenção. Revista Ciência e Saúde Coletiva, 10 (1), 143-149.

Bye, E. K. (2007). Alcohol and violence: use of possible confounders in a time-series analysis. Addiction, 102 (3), 369-376.

Day, V., Telles, L., Zoratto, P., Azambuja, M., Machado, D., Silveira, M., et al. (2003). Violência doméstica e suas manifestações. Revista de Psiquiatria, 25 (1), 9-21.

Departamento de Informação do Sistema Único de Saúde (2009). Caderno de informações de saúde. Recuperado em julho 10, 2009, disponível em <http://www2.datasus. gov.br/DATASUS/index.php?area=02>.

Espídola, C. R., \& Blay, S. L. (2007). Prevalência de maus-tratos na terceira idade: revisão sistemática. Revista de Saúde Pública, 4 (2), 301-306.

Haggård-Grann, U., Hallqvist, J., Långström, N., \& Möller, J. (2006). The role of alcohol and drugs in triggering criminal violence: a case-crossover study. Addiction, 101 (1), 100-108.

Instituto Brasileiro de Geografia e Estatística. (2002). Perfil dos idosos responsáveis pelo domicílio. Pesquisa nacional por amostragem domiciliar. Recuperado em julho 15, 2009, disponível em <http://www.ibge.gov.br>.

Irigaray, T. Q., \& Trentini, C. M. (2009). Qualidade de vida em idosas: a importância da dimensão subjetiva. Estudos de Psicologia (Campinas), 26 (3), 297-304. doi: 10.1590/S0 103-166X2009000300003.

Krüger, H. R. (1993). Crenças e sistemas de crenças. Arquivos Brasileiros de Psicologia, 45 (1), 3-15.

Krüger, H. R. (1995). Psicologia das crenças: perspectivas teóricas. Tese de concurso para professor titular não-publicada, Departamento de Psicologia Social Institucional, Universidade Estadual do Rio de Janeiro.

Machado, L., \& Queiroz, Z. (2002). Negligência e maus-tratos. In E. V. Freitas, L. Py, L. Cançado \& M. Gonzoni (Orgs.). Tratado de geriatria e gerontologia (pp.791-797). Rio de Janeiro: Guanabara Koogan.

Minayo, M. C. S. (2003). Violência contra idosos: relevância para um velho problema. Cadernos de Saúde Pública, 19 (3), 783-791.

Minayo, M. C. S. (2006). Violência e saúde. Rio de Janeiro: Fiocruz. 
Moraes, C. L., Apratto Júnior, P. C., \& Reichenheim, M. E. (2008). Rompendo o silêncio e suas barreiras: um inquérito do-miciliar sobre a violência doméstica contra idosos em áreas de abragência do Programa Médico de Família de Niterói, Rio de Janeiro, Brasil. Cadernos de Saúde Pública, 24 (10), 2289-2300.

Noto, A. R., Fonseca, A. M., Silva, E. A., \& Galduròz, J. C. F. (2004). Violência domiciliar associada ao consumo de bebidas alcoólicas e de outras drogas: um levantamento no estado de São Paulo. Jornal Brasileiro de Dependência Química, 5 (1), 9-17.

Organização Mundial da Saúde. (2002). Relatório mundial sobre violência e saúde. Genebra: OMS.

Pasinato, M. T., Camarano, A. M., \& Machado, L. (2006). Idosos vítimas de maus-tratos domésticos: estudo exploratório das informações dos serviços de denúncia (Texto para discussão n. 1200). Rio de Janeiro: IPEA.

Porto, l., \& Koller, S. H. (2006). Violência familiar contra pessoa idosa. Interações, 12 (22), 105-142.

Rokeach, M. (1981). Crenças, atitudes evalores. Rio de Janeiro: Interciência.

Sanches, A. R. A., Lebrão, M. L., \& Duarte, Y. A. O. (2008). Violência contra idosos: uma questão nova? Saúde e Sociedade, 17 (3), 90-100.
Santos, A. P., Silva, C. A., Carvalho, L. S., \& Menezes, M. R. (2007). A construção da violência doméstica contra idosos. Revista Brasileira de Geriatria e Gerontologia, 10 (1), 129-140.

Silvestre, J. A., \& Costa Neto, M. M. (2003). Abordagem do idoso em programas de saúde da família. Cadernos de Saúde Pública, 19 (3), 839-847.

Souza, A. S., Meira, E. C., Neri, I. G., Silva, J. A., \& Gonçalves, L. T. (2004). Fatores de risco de maus-tratos ao idoso/ cuidador em convivência intrafamiliar. Textos sobre Envelhecimento, 7 (2), 63-85.

Souza, E. R., Ribeiro, A. P., Atie, S., Souza, A. C., \& Marques, C. C. (2008). Rede de proteção aos idosos do Rio de Janeiro: um direito a ser conquistado. Ciências e Saúde Coletiva, 13 (4), 1153-1163.

Souza, J. A. V., Freitas, M. C., \& Queiroz, T. A. (2007). Violência contra os idosos: análise documental. Revista Brasileira de Enfermagem, 60 (3), 268-272.

Recebido em: 30/1/2010

Versão final reapresentada em: 26/4/2011

Aprovado em: 19/12/2011 